\title{
Completed Absorption of Coronavirus Disease 2019 (COVID-19) Pneumonia Lesions: A Preliminary Study
}

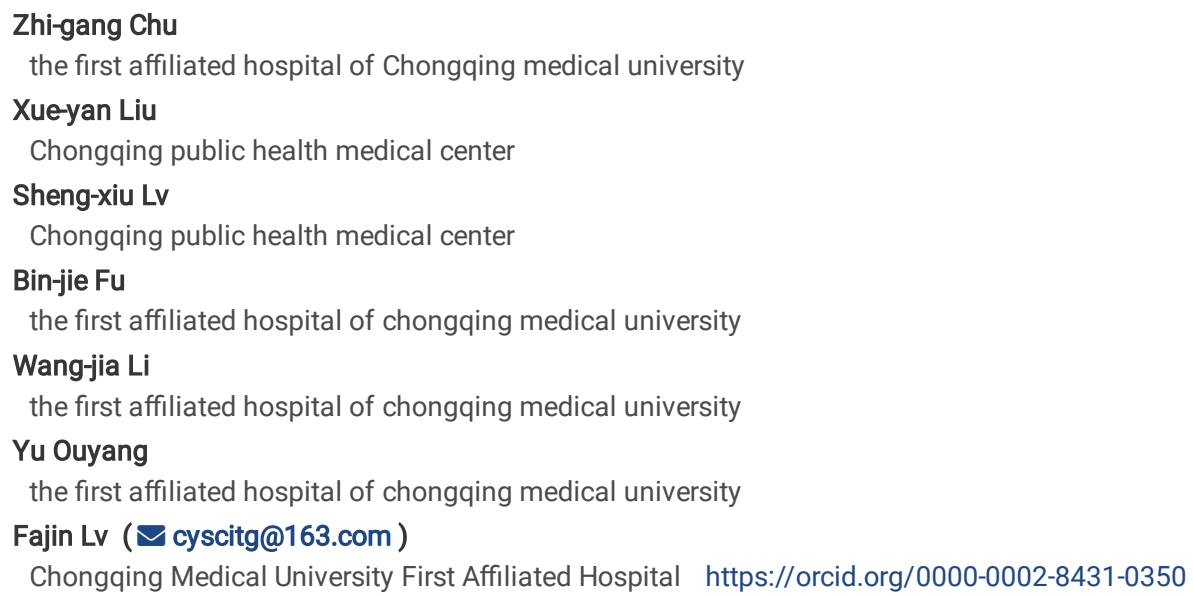




\section{Abstract}

Background Complete absorption of coronavirus disease 2019 (COVID-19) pneumonia in a short term was not detailedly reported. We aimed to investigate the clinical and imaging characteristics of COVID-19 patients with complete absorption of pulmonary lesions.

Methods Retrospectively collected the clinical and chest CT data of 224 patients with COVID-19 in one regional medical center. Currently, pulmonary lesions in 37 patients were completely absorbed. The clinical manifestations, laboratory examinations, and CT findings of lesions for these patients were summarized.

Results Among the 37 patients (age, $39.0 \pm 12.4$ [14 - 63] years, 20 males), disease in 36 (97.3\%) was mild and in 1 (3.7\%) was from severe to mild. The most common symptoms were cough $(24 / 37,64.9 \%)$ and fever $(23 / 37,62.2 \%)$. Their laboratory indicators at admission were usually normal, while the white blood cell and neutrophil count significantly increased at discharge $(p=0.004, p=0.006)$. On initial CT images, all patients had various pulmonary lesions (mean involved lobes:2.8 \pm 1.5 , range:1-5; mean involved segments: $6.6 \pm 4.3$, range: $1-16)$, which mainly manifested as multiple patchy and or spherical ground glass opacities (GGOs) (30/37, 81.1\%) with fibrous strips (19/30, 63.3\%) or consolidation (11/30, 36.7\%). After treatment, lesions in most (33/37, 89.2\%) patients were continuously absorbed. At discharge, previous lesions were mostly absorbed in 11 patients $(11 / 37,29.7 \%)$, the main residues were GGOs (24/37, $64.9 \%)$, followed by fibrous strips $(13 / 37,35.1 \%)$. On the latest CT, all the pulmonary lesions were completely absorbed, the duration of lesions was $31.6 \pm$ 11.4 days (range: 5 - 50 days).

Conclusion The pulmonary lesions in some mild COVID-19 patients (generally with normal laboratory indicators at admission, GGOs as the main manifestation on initial CT, and representation of continuous absorption after treatment) could be completely absorbed with a mean duration of 31.6 days.

\section{Background}

Since December 2019, the occurrence of unexplained pneumonia was first reported in Wuhan City, Hubei Province, China [1]. Virus detected and isolated from the patient's respiratory epithelial cells was named Severe Acute Respiratory Syndrome Coronavirus-2 (SARS-CoV-2). Subsequently, pneumonia caused by the infection of this virus was called Coronavirus 2019 (COVID-19). This virus is highly contagious, approximately with a basic regeneration number of 2.2 [2]. Currently, the COVID-19 has spread worldwide and was declared a global health emergency by WHO. As of April 20, 2020, more than 2 million cases have been confirmed in more than 200 countries and regions [3].

The SARS-CoV-2 mainly involves the respiratory system. Chest CT has a high sensitivity for diagnosing COVID-19 pneumonia [4], and plays an important role in the follow-up of COVID-19 patients. Due to positive findings on CT scans could be found before appearance of symptoms [5], thus chest CT also can be used for screening patients with negative reverse transcriptase polymerase chain reaction (RT-PCR) results in the early stage [6,7]. Several studies on COVID-19 imaging have described the CT features of this disease and the evolution of the lesions [8-12]. However, at present, there was no systematical reports on the outcome of this disease except a study reporting only one patient with complete absorption of the pulmonary lesion on the 31 st day after the onset of symptoms [13].

In view of this, whether complete absorption of COVID-19 pneumonia is common, how long is the duration of lesions, and the clinical and imaging characteristics of those patients with completely absorbed lesions are unknown. Our hospital as one designated medical center for treating COVID-19 patients, a total of 224 patients were cured and discharged from hospital without death. After discharge, follow-up CT scans were still performed in our hospital. Currently, it was found that some mild patients' pulmonary lesions have been completely absorbed on repeat CT. This study aims to investigate their clinical and imaging characteristics for better understanding the outcome of this disease.

\section{Methods}

This retrospective study used non-identifiable patient data for analysis and was approved by the institutional review board. Therefore, the requirement for informed consent was waived. The collection and analysis of anonymous data pose no potential risk to patients.

\section{Patients}

A total of 224 patients with COVID-19 confirmed by RT-PCR test were admitted to our hospital from January 24, 2020 to March 27, 2020. Inclusion criteria: (1) there were pulmonary lesions on initial chest CT; (2) the patients' clinical data and laboratory examinations at admission were complete. Exclusion criteria: (1) the pulmonary lesions did not change or were absorbed incompletely on the latest repeat CT; (2) the interval between initial chest CT scan and hospitalization was more than 1 day.

\section{Treatment and discharge Criteria}

The treatment and discharge standards for COVID-19 patients were comply with the "Diagnosis and Treatment Program of Coronavirus Disease 2019 (Trial Seventh Edition)" [14]. Discharge criteria were: (1) the body temperature returned to normal for more than 3 days, (2) respiratory symptoms improved significantly, (3) chest imaging examinations showed significant improvement of acute exudative lesions, (4) two consecutive nucleic acid tests on respiratory tract specimens were negative (the interval of two test must be at least 24 hours).

\section{CT Protocol}

All patients underwent a non-contrast CT upon admission to the hospital. The initial and repeat chest CT scans were performed using Aquilion ONE (Toshiba Medical Systems, Tokyo, Japan) and Neusoft on-board 32-row CT scanner with the following parameters: tube voltage, $120 \mathrm{kV}$; automatic tube current modulation; beam pitch,1.2/1.438; detector collimation, 0.625/0.6 mm; rotation time, $0.5 \mathrm{~s}$; matrix size, $512 \times 512$; and section thickness and interval, 5.0 and 
$5.0 \mathrm{~mm}$, respectively. All patients were in a supine position and scanned from the thoracic inlet to the lung base at the end of inspiration. CT images were reconstructed using a medium sharp reconstruction algorithm with a section thickness of $1 \mathrm{~mm}$.

\section{Imaging analysis}

Two senior radiologists (Lv and Chu), specialized in chest imaging read all the patients' first and follow-up CT images independently. The first step is to determine whether the pulmonary lesions in the initial chest CT scan are related to COVID-19. Subsequently, the follow-up CT data were reviewed and compared with the initial result for determining the changes and residues of lesions. The evaluation indicators are as follows: a. distribution of initial lesions (unilateral lung or bilateral lungs, involved lobes and segments); b. involved scope (the number of involved lobes and segments); c. the shape of lesions (round or patchy); d. density (ground glass opacity or consolidation); e. internal features (fibrous strips or consolidation); f. the changes of lesions one repeat CT (progression or directly absorption), g. outcome of lesions on latest CT scan (no change, incompletely absorption, complete absorption). In addition, pleural effusion and mediastinal lymphadenopathy were also evaluated. The result was based on the opinions of the two radiologist and reached an agreement.

\section{Clinical Features}

Clinical and laboratory data of the patients were collected by one radiologist (X.Y. L). Clinical data, including age, gender, clinical type, initial symptoms, length of hospitalization, numbers of scans, and the interval between the initial and latest CT scans were recorded. Laboratory findings such as white blood cell count, neutrophil count and percentage, lymphocyte count and percentage, C-reactive protein, erythrocyte sedimentation rate, and lactate dehydrogenase, were also recorded. These laboratory tests were performed at admission and discharge.

\section{Statistical Analysis}

Statistical analysis was performed using SPSS (version 24; IBM, New York, USA). Continuous variables were expressed as mean \pm standard deviation (minimum-maximum), and categorical variables were expressed as numbers and percentages. Independent sample $t$ test (normal distribution or variance) or Mann-Whitney U test (non-normal distribution or variance) were used for statistical analysis. A p value $<0.05$ was considered statistically significant.

\section{Results}

\section{Clinical characteristics}

Among the 224 patients with COVID-19, 37 (16.5\%) cases were finally included in this study. The clinical characteristics of patients are shown in Table 1. The most common clinical manifestations were cough (64.9\%) and fever (62.2\%). The disease in most patients were mild ( $97.3 \%)$, while that in the other one changed from severe into mild during treatment.

\begin{tabular}{|c|c|}
\hline Parameters & Value \\
\hline \multicolumn{2}{|l|}{ Gender } \\
\hline Male & $20(54.1)$ \\
\hline Female & $17(45.9)$ \\
\hline Age (years) & $39.0 \pm 12.4(14-63)$ \\
\hline \multicolumn{2}{|l|}{ Time of onset of symptoms } \\
\hline Average (days) & $4.6 \pm 3.0(1-12)$ \\
\hline$\geq 7$ days & $29(78.4)$ \\
\hline ૧7days & $6(16.2)$ \\
\hline \multicolumn{2}{|l|}{ Exposure History } \\
\hline Recently travel to main epidemic area & $10(27.0)$ \\
\hline Exposure to infected patients & $19(51.4)$ \\
\hline Contacting with people from epidemic area & $4(10.8)$ \\
\hline Unknow & $4(10.8)$ \\
\hline \multicolumn{2}{|l|}{ Clinical type } \\
\hline Mild & $36(97.3)$ \\
\hline Severe & $1(2.7)$ \\
\hline \multicolumn{2}{|l|}{ Maximum body temperature $\left({ }^{\circ} \mathrm{C}\right)$} \\
\hline $37.3-38.0$ & $17(45.9)$ \\
\hline $38.1-39.0$ & $6(16.2)$ \\
\hline$>39.0$ & $0(0)$ \\
\hline \multicolumn{2}{|l|}{ Clinical manifestations } \\
\hline Fever & $23(62.2)$ \\
\hline Cough & $24(64.9)$ \\
\hline Sputum & $11(29.7)$ \\
\hline Headache & 7 (18.9) \\
\hline Sore Throat & 7 (18.9) \\
\hline Fatigue & $6(16.2)$ \\
\hline Diarrhea & $6(16.2)$ \\
\hline Muscle Soreness & 4 (10.8) \\
\hline Chills & $3(8.1)$ \\
\hline Asymptomatic & $2(5.4)$ \\
\hline
\end{tabular}

Data are expressed as mean \pm standard deviation (range) or $\mathrm{n}(\%)$.

\section{Laboratory indicators}


The laboratory characteristics of the patients at admission and discharge are listed in Table 2. At admission, laboratory results in most patients were in normal limits, and most of those abnormal indicators recovered at discharge. The white blood cell count and neutrophil count at discharge were higher than those at admission ( $5.99 \pm 1.84$ vs. $4.83 \pm 1.50, p=0.004 ; 3.67 \pm 1.43$ vs. $2.81 \pm 1.15, p=0.006$ ), while lactate dehydrogenase was significantly decreased at discharge compared with that at admission $(164.1 \pm 29.46$ vs. $220.8 \pm 90.64, p=0.001)$.

Table 2: The laboratory characteristics of patients

\begin{tabular}{|c|c|c|}
\hline Laboratory examinations & At admission & At discharge \\
\hline White blood cell count $(\mathrm{G} / \mathrm{L})$ & & \\
\hline Normal / decreased & $33(89.2)$ / 4 (10.8) & $34(91.9) / 3(8.1)$ \\
\hline Neutrophil count (G/L) & & \\
\hline $\begin{array}{l}\text { Normal / increased/ decreased } \\
\text { Neutrophil percentage (\%) }\end{array}$ & $32(86.5) / 0(0) / 5(13.5)$ & $33(89.2) / 2(5.4) / 2(5.4)$ \\
\hline $\begin{array}{l}\text { Normal / increased/ decreased } \\
\text { Lymphocyte count (G/L }\end{array}$ & $32(86.5) / 2(5.4) / 3(8.1)$ & $36(97.3) / 0(0) / 1(2.7)$ \\
\hline $\begin{array}{l}\text { Normal / increased/ decreased } \\
\text { Lymphocyte percentage (\%) }\end{array}$ & $32(86.5) / 0(0) / 5(13.5)$ & $33(89.2) / 1(2.7) / 3(8.1)$ \\
\hline $\begin{array}{l}\text { Normal / increased/ decreased } \\
\text { C-reactive protein }(\mathrm{mg} / \mathrm{L})\end{array}$ & $32(86.5) / 2(5.4) / 3(8.1)$ & $35(94.6) / 0(0) / 2(5.4)$ \\
\hline Normal / increased & $20^{\mathrm{a}}(66.7) / 10^{\mathrm{a}}(33.3)$ & $18^{\mathrm{b}}(85.7) / 3^{\mathrm{b}}(14.3)$ \\
\hline $\begin{array}{l}\text { Lactate dehydrogenase (U/L) } \\
\text { Normal / increased }\end{array}$ & $27(73.0) / 10(27.0)$ & $35^{c}(100.0) / 0^{c}(0.0)$ \\
\hline CD3 $(/ \mu \mathrm{L})$ & & \\
\hline $\begin{array}{l}\text { Normal / decreased } \\
\text { CD4 }(/ \mu \mathrm{L})\end{array}$ & $28(75.7) / 9(24.3)$ & $8^{\mathrm{d}}(80.0) / 2^{\mathrm{d}}(20.0)$ \\
\hline $\begin{array}{l}\text { Normal / decreased } \\
\text { CD8 }(/ \mu L)\end{array}$ & $25(67.6) / 12(32.4)$ & $8^{\mathrm{d}}(80.0) / 2^{\mathrm{d}}(20.0)$ \\
\hline Normal / decreased & $29(78.4) / 8(21.6)$ & $9^{d}(90.0) / 1^{d}(10.0)$ \\
\hline
\end{tabular}

Notes: ${ }^{\mathrm{a}}, \mathrm{n}=30 ;{ }^{\mathrm{b}}, \mathrm{n}=21{ }^{\mathrm{c}}, \mathrm{n}=35{ }^{\mathrm{d}}, \mathrm{n}=10$

Data are expressed as $n(\%)$.

In 37 patients, the average number of nucleic acid tests at the time of first turning negative and the total number of nucleic acid tests during hospitalization were $4.3 \pm 4.1$ ( 1 - 15) times and $5.7 \pm 4.2$ (range: 2 - 16) times, respectively. The mean time between the first positive nucleic acid test and the first negative nucleic acid test was $17.1 \pm 8.8$ (range: 5 - 36) days.

\section{CT features}

At admission, all the 37 patients showed various pulmonary lesions on chest CT, their distribution and extent are shown in Table 3. The mean numbers of involved lobes and segments were $2.8 \pm 1.5$ (1-5) and $6.6 \pm 4.3$ (1-16), respectively. On CT images, pulmonary lesions manifested as multiple patchy and/or spherical GGOs in $30(81.1 \%)$ cases $(11$ [36.7\%] cases with little consolidation and 19 [63.3\%] cases with fibrous strips), multiple patchy consolidations with little GGO in 4 cases (10.8\%); localized patchy GGOs with fibrous strips 3 (8.1\%) cases. Besides these lesions, calcification, small solid nodules, fibrous strips, and bullae were found in $5(13.5 \%)$ cases. No pleural effusion or mediastinal and hilar lymphadenopathy was detected in all patients.

Table 3. Distribution and extent of pulmonary lesions at admission

\begin{tabular}{ll}
\hline Distribution and extent & Value \\
\hline Bilateral lung involvement & $27(73.0)$ \\
Unilateral lung involvement & $10(27.0)$ \\
Number of involved lobes & \\
1 & $10(27.0)$ \\
2 & $6(16.2)$ \\
3 & $7(18.9)$ \\
4 & $8(21.6)$ \\
5 & $6(16.2)$ \\
Frequency of involved lobes & \\
Right upper lobe & $14(37.8)$ \\
Right middle lobe & $14(37.8)$ \\
Right lower lobe & $32(86.5)$ \\
Left upper lobe & $17(45.9)$ \\
Left lower lobe & $28(75.7)$ \\
Number of involved segments & \\
1 & $2(5.4)$ \\
$2-4$ & $13(35.1)$ \\
$5-7$ & $9(24.3)$ \\
8-10 & $5(13.5)$ \\
$\square 11$ & $8(21.6)$ \\
\hline
\end{tabular}

Notes: data are expressed as $\mathrm{n}$ (percentage).

The mean duration of hospitalization for the patients was $18.7 \pm 8.2$ days (range: 6 - 37 days). On repeat CT scans during treatment, lesions in 33 ( $89.2 \%$ ) patients were continuously absorbed and in 4 cases (10.8\%) showed a mild progression before absorption. At discharge, among the 37 patients, lesions in 11 $(29.7 \%)$ cases were almost absorbed, in $25(67.6 \%)$ showed residues of pure GGOs or GGOs with consolidation or fibrous strips, and in 2 (5.4\%) cases showed only few fibrous strips. The mean involved lobes and segments were $1.6 \pm 1.6(1-5)$ and $3.8 \pm 4.1(1-14)$ on the latest CT before discharge.

After discharge, all the pulmonary lesions were completely absorbed on the latest CT (Fig1, Fig2), the duration of lesions from occurrence to disappearance was $31.6 \pm 11.4$ days (range: 5-50 days). Among different lesions, duration of GGO was the longest (mean: $29.8 \pm 12.1$ days, range: 5.0 - 50.0 ), which was 
significantly longer than that of fibrous strips (mean: $23.8 \pm 9.5$ days, range: $12.0-44.0)(p=0.046)$ and consolidation (mean: $9.4 \pm 3.6$ days, range: $4.0-15.0)$ $(p=0.000)$.

\section{Discussion}

Coronaviridae viruses include six previously known human infectious coronaviruses, which are widely distributed in humans and other mammals. SARS-CoV-2 is the seventh member of the coronaviridae family known to infect humans. It is significantly different from the genetic characteristics of SARS-CoV and MERS-CoV [14]. In view of the current epidemic situation, SARS-CoV-2 is highly contagious, which posed a major threat to public health. However, most COVID19 patients in our hospital showed a good prognosis, and pulmonary lesions in some cases were found to be completely absorbed in a short period of time. Thus, it is necessary to summarize the clinical and imaging characteristics of such patients for better understanding this disease.

For SARS-CoV-2, the population is generally susceptible, and usually the elderly or those with underlying diseases are more severe after infection [15]. In the present study, the average age of patients was 39 years old, which was lower than that of general patients in previous studies [12].It indicates that younger patients may have a better prognosis because of relative stronger immunity and less basic diseases. Laboratory examinations also revealed routine hematological and inflammatory indicators in most patients were normal or mildly abnormal at admission and those abnormalities usually return to normal before discharge. Additionally, the disease in most patients was mild, indicating lung injury was not serious [9], which may be also closely related to their better prognosis. Therefore, the pulmonary lesions in most patients may be completely absorbed finally because of the higher percentage of mild disease in infected cases [16].

Besides the clinical and laboratory characteristics, CT manifestations of pulmonary lesions and their changes also showed some characteristics during treatment. In this study, most lesions manifested as GGOs without significant consolidation at admission, and their extent was less than that in severe patients reported in previous study [17]. After accepting treatment, lesions in most patients showed continuous absorption, which was different from those showing progression before absorption $[8,11]$. These characteristics indicated the pulmonary damage was not as serious as that caused by significant consolidation [18], and the disease was well controlled.

At present, the relationship between occurrence of fibrous strips and prognosis of COVID-19 patients is still controversial. Zhou S et al. [11] believed that the lesions could lead to pulmonary interstitial fibrosis after the appearance of fibrosis strips, indicating a poor prognosis. While Pan $Y$ et al. [5] reported that the appearance of fibrous strips suggested improvement of disease and good prognosis. In the present study, the newly developed fibrous strips were commonly detected in pulmonary lesions during treatment, and most of which were completely absorbed eventually before GGO. Therefore, the appearance of fibrous strips may be a common manifestation in later stage of disease and it does not necessarily indicate a poor prognosis.

The time from the onset of lesions to complete absorption varied among individuals with a large range. Absorption of GGOs was significantly slower than that of fibrous strips or consolidation. The pathological basis for GGO caused by SARS-Cov-2 included alveolar injury, edema, and exudation with alveolar epithelial cell proliferation and inflammatory infiltration [19]. Therefore, the duration of GGO or pulmonary lesions was closely related to the degree of pulmonary injury. Longer duration of lesions represented more serious injury.

There are the three limitations in this study. First, all the patients had pulmonary lesions on initial CT scan and there were intervals between two repeat CT scans, thus the exact duration of lesions could not be acquired. Second, we did not compare the patients with complete and incomplete absorption of lesions at the same period. The cases in control group cannot be well matched because the duration of lesions significantly varied among patients. Third, disease in most patients in the present study was mild. The patients with severe disease were not included because they still had residues on the latest CT scan though the lesions were continuously absorbed. Thus, further studied is needed.

\section{Conclusions}

In conclusion, pulmonary lesions in some patients with mild COVID-19 pneumonia could be completely absorbed in a short term, with a mean duration of 31.6 days and a large range. These patients are usually younger and have normal laboratory indicators at admission. Their lesions on initial CT are mainly manifested as GGOs, and which frequently be absorbed continuously after treatment.

\section{Abbreviations}

COVID-19: coronavirus disease 2019; GGOs: ground-glass opacities; RT-PCR: reverse transcriptase polymerase chain reaction.

\section{Declarations}

Ethics approval and consent to participate: This retrospective study was approved by the medical ethical committee of the First Affiliated Hospital of Chongqing Medical University and the requirement for informed consent was waived.

Consent for publication: Not applicable.

Availability of data and materials: The datasets used and/or analyzed during the current study are available from the corresponding author on reasonable request.

Funding: None. 
Competing interests: The authors declare that they have no competing interests.

\section{Author information}

1.The First Affiliated Hospital of Chongqing Medical University, 1\# Youyi Road, Yuanjiagang, Yuzhong district, Chongqing, 400016, People's Republic of China.

Zhi-gang Chu, Bin-jie Fu, Wang-jia Li, Yu Ouyang, Fa-jin Lv.

2.ChongQing Public Health Medical Center, Chongqing, 400016, People's Republic of China.

Xue-yan Liu, Sheng-xiu Lv.

Author contributions: ZGC and XYL contributed to the conceptualization, writing-original draft preparation, writing-review and editing, supervision and visualization. FJL contributed to the writing-review, editing and supervision. SXL, BJF, WJL and YOY contributed to the conceptualization. All authors have approved the final version of the work.

Corresponding authors: Correspondence to Fajin Lv.

Acknowledgments: Not applicable.

\section{References}

1. Novel Coronavirus - China. World Health Organization. https://www.who.int/csr/don/12-january-2020-novel-coronavirus-china/en/.

2. Li Q, Guan X, Wu P, et al. Early Transmission Dynamics in Wuhan, China, of Novel Coronavirus-Infected Pneumonia. N Engl J Med 2020. 26;382(13):11991207.

3. Johns Hopkins Whiting School of Engineering website. Coronavirus COVID-19 global cases.gisanddata.maps.arcgis.com/apps/opsdashboard/index.https://www.arcgis.com/apps/opsdashboard/index.html\#/bda7594740fd40299423467b

4. Ai T, Yang Z, Hou H, et al. Correlation of Chest CT and RT-PCR Testing in Coronavirus Disease 2019 (COVID-19) in China: A Report of 1014 Cases. Radiology. 2020 Feb 26:200642. [Epub ahead of print]

5. Pan Y, Guan H, Zhou S, et al. Initial CT findings and temporal changes in patients with the novel coronavirus pneumonia (2019-nCoV): a study of 63 patients in Wuhan, China. Eur Radiol 2020 Feb 13 [Epub ahead of print]

6. Huang P, Liu T, Huang L, et al. Use of chest CT in combination with negative RT-PCR assay for the 2019 novel coronavirus but high clinical suspicion. Radiology 2020 Feb 12 [Epub ahead of print]

7. Xie X, Zhong Z, Zhao W, et al. Chest CT for typical 2019-nCoV pneumonia: relationship to negative RT-PCR testing. Radiology 2020 Feb 12 [Epub ahead of print]

8. Wang Y, Dong C, Hu Y, et al. Temporal Changes of CT Findings in 90 Patients with COVID-19 Pneumonia: A Longitudinal Study. Radiology. 2020 Mar 19:200843. [Epub ahead of print]

9. Liu KC, Xu P, Lv WF, et al. CT manifestations of coronavirus disease-2019: A retrospective analysis of 73 cases by disease severity. Eur J Radiol. 2020 Mar 12; 126:108941. [Epub ahead of print]

10. Shi H, Han X, Jiang N, et al. Radiological findings from 81 patients with COVID-19 pneumonia in Wuhan, China: a descriptive study. Lancet Infect Dis. 2020 Feb 24. pii: S1473-3099(20)30086-4. [Epub ahead of print]

11. Pan F, Ye T, Sun P, et al. Time Course of Lung Changes on Chest CT During Recovery From 2019 Novel Coronavirus (COVID-19) Pneumonia. Radiology 2020 Feb 13:200370. doi: 10.1148/ radiol.2020200370. [Epub ahead of print]

12. Zhou S, Wang Y, Zhu T, et al. CT Features of Coronavirus Disease 2019 (COVID-19) Pneumonia in 62 Patients in Wuhan, China. AJR Am J Roentgenol. 2020 Mar 5:1-8. [Epub ahead of print]

13. Shi H, Han X, Zheng C. Evolution of CT Manifestations in a Patient Recovered from 2019 Novel Coronavirus (2019-nCoV) Pneumonia in Wuhan, China. Radiology. 2020;295(1):20.

14. National Health Commission of the People's Republic of China. The guidlines for the diagnosis and treatment of 2019-nCoV pneumonia (the 7th edition). http://www.nhc.gov.cn/yzygj/s7653p/202003/46c9294a7dfe4cef80dc7f5912eb1989.shtml?spm=C73544894212.P59511941341.0.0

15. Xu X, Wu X, Jiang X, et al. Clinical findings in a group of patients infected with the 2019 novel coronavirus (SARS-Cov-2) outside of Wuhan, China: retrospective case series. BMJ 2020:m606.

16. Li K, Wu J, Wu F, et al. The Clinical and Chest CT Features Associated with Severe and Critical COVID-19 Pneumonia. Invest Radiol. 2020 Feb 29. doi: 10.1097/RLI.000000000 0000672. [Epub ahead of print]

17. Rodriguez-Morales AJ, Cardona-Ospina JA, Gutiérrez-Ocampo E, et al. Clinical, laboratory and imaging features of COVID-19: A systematic review and meta-analysis [published online ahead of print, 2020 Mar 13]. Travel Med Infect Dis. 2020; 101623.

18. Bernheim A, Mei X, Huang M, et al. Chest CT Findings in Coronavirus Disease-19 (COVID-19): Relationship to Duration of Infection [published online ahead of print, 2020 Feb 20]. Radiology. 2020;200463.

19. Tian S, Hu W, Niu L, Liu H, Xu H, Xiao SY. Pulmonary Pathology of Early-Phase 2019 Novel Coronavirus (COVID-19) Pneumonia in Two Patients With Lung Cancer. J Thorac Oncol. 2020. [published online ahead of print, 2020 Feb 28] 


\section{Figures}
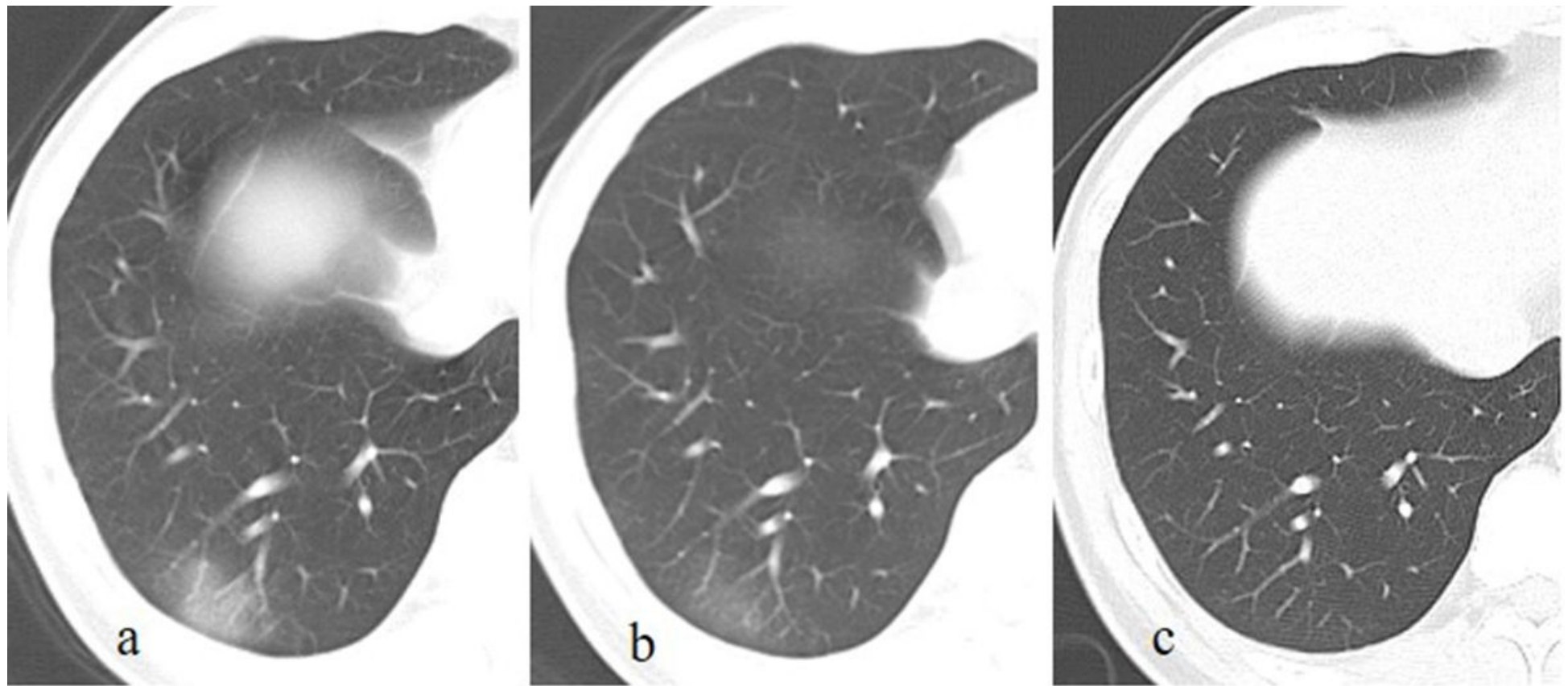

\section{Figure 1}

A patient with a history of travelling to Wuhan recently, presents with fever and cough for 13 days. The initial chest CT at the first day after admission shows a patchy GGO in the right lower lobe (a). Subsequently (5 days later), this lesion is directly absorbed with decrease of extent and density (b). On the latest CT scan (18 days later), the lesion is completely absorbed (c).
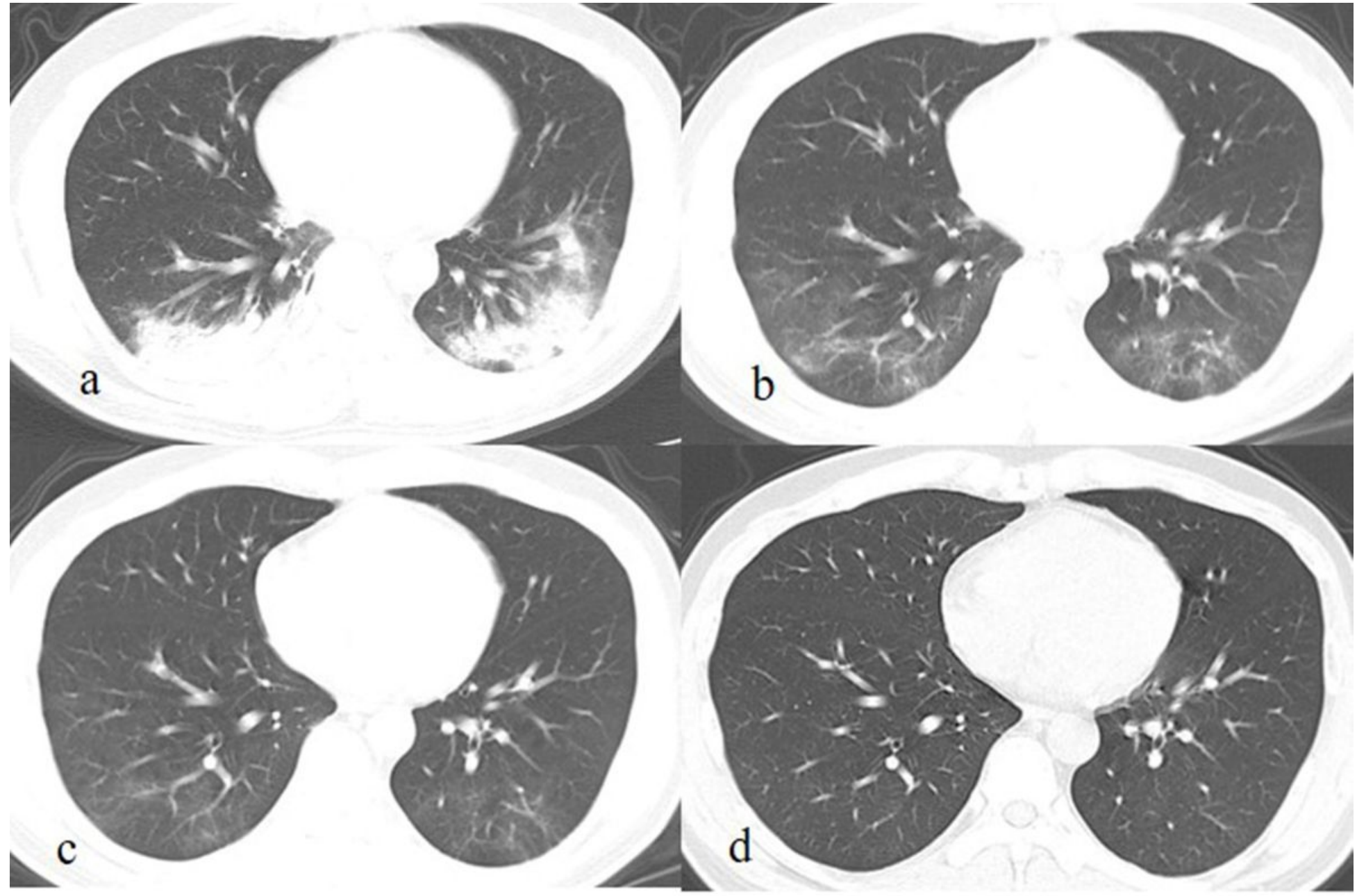
Figure 2

A patient exposed to COVID-19 presents with fever, cough, and fatigue for 7 days. The initial chest CT at the first day after admission shows patchy consolidations and GGO in the left and right lower lobes (a). Subsequently (7 and 13 days later), the lesions are continuously absorbed with decrease of extent and density, and fibrous strips are detected in lesions (b, c). On the latest CT scan (36 days later), the lesion is completely absorbed GGO (c). 